\title{
STATE VARIANCE CONSTRAINED FUZZY CONTROL VIA OBSERVER-BASED FUZZY CONTROLLERS
}

\author{
Wen-Jer Chang \\ Associate Professor, Department of Civil Engineering, National Taipei University of Technology, No. 1, Sec. 3, \\ ZhongXiao E. Rd., Taipei, Taiwan 10608, R.O.C., wjchang@mail.ntou.edu.tw \\ Balasingam Yeh \\ Master, Department of Marine Engineering, National Taiwan Ocean University, Keelung, Taiwan 202, R.O.C. \\ Yu-Teh Meng \\ Student, Department of Marine Engineering, National Taiwan Ocean University, Keelung, Taiwan 202, R.O.C.
}

Follow this and additional works at: https://jmstt.ntou.edu.tw/journal

Part of the Engineering Commons

\section{Recommended Citation}

Chang, Wen-Jer; Yeh, Balasingam; and Meng, Yu-Teh (2006) "STATE VARIANCE CONSTRAINED FUZZY CONTROL VIA OBSERVER-BASED FUZZY CONTROLLERS," Journal of Marine Science and Technology. Vol. 14: Iss. 1, Article 5. DOI: $10.51400 / 2709-6998.2078$

Available at: https://jmstt.ntou.edu.tw/journal/vol14/iss1/5

This Research Article is brought to you for free and open access by Journal of Marine Science and Technology. It has been accepted for inclusion in Journal of Marine Science and Technology by an authorized editor of Journal of Marine Science and Technology. 
STATE VARIANCE CONSTRAINED FUZZY CONTROL VIA OBSERVER-BASED FUZZY CONTROLLERS

Acknowledgements

This work was supported by the National Science Council of the Republic of China under contract NSC932218-E-019-002. 


\title{
STATE VARIANCE CONSTRAINED FUZZY CONTROL VIA OBSERVER-BASED FUZZY CONTROLLERS
}

\author{
Wen-Jer Chang*, Yi-Lin Yeh**, and Yu-Teh Meng***
}

Key words: takagi-sugeno fuzzy models, state variance constraints, linear matrix inequalities (LMI).

\begin{abstract}
In this paper, a state variance constrained design method is developed to deal with the observer-based fuzzy control problem for continuous Takagi-Sugeno (T-S) fuzzy stochastic models. The stability problem considered in this paper is a standard feasibility problem with several Linear Matrix Inequalities (LMIs), which can be solved numerically using the interior-point method. The purpose of this paper aims at finding observer-based fuzzy controllers such that the closed-loop system is asymptotically stable and the state variance performance constraints are satisfied. Finally, a numerical example is included to demonstrate the effectiveness of the proposed controller design method.
\end{abstract}

\section{INTRODUCTION}

The fuzzy logic has been applied extensively in the areas of industrial system and consumer products. It has emerged in turn to tackle the control problems where the plants are poorly modeled by mathematics. However, the heuristics-based [14] approach in the early days of fuzzy logic control lack the formula and system design methodology that guarantees the basic requirements such as stability and acceptable performance. The theoretic researches on the issue are conducted actively by many theorists since the T-S fuzzy model [19] is emerged.

The T-S fuzzy model is described by a set of IFTHEN rules. These rules locally represent linear inputoutput relations of nonlinear systems. The stability analysis and controller design for T-S type fuzzy sys-

Paper Submitted 01/19/05, Accepted 07/28/05. Author for Correspondence: Wen-Jer Chang. E-mail: wjchang@mail.ntou.edu.tw.

* Professor, Department of Marine Engineering, National Taiwan Ocean University, Keelung, Taiwan 202, R.O.C.

**Master, Department of Marine Engineering, National Taiwan Ocean University, Keelung, Taiwan 202, R.O.C.

***Student, Department of Marine Engineering, National Taiwan Ocean University, Keelung, Taiwan 202, R.O.C. tems are discussed in $[2-4,10,19,25,26]$. The controller design is carried out based on the fuzzy model via socalled Parallel Distributed Compensation (PDC) concept $[2-4,10,25,26]$. Based on PDC concept [2-4, 10, $25,26]$, Lyapunov theory is the main tool for designers to deal with the stability analysis and synthesis of T-S fuzzy models. The advantage of the PDC concept is to design linear feedback gains for each local linear model and let the overall system input can be blended by these linear feedback gains.

In control theory, the performance constraint usually plays an important objective in addition to the stability requirement. For example, the state variance constraints [5, 7-9] are important performance requirements for the stochastic control systems. In this paper, we not only consider the stability of nonlinear systems, but also consider the state variance constraints. It is shown that this state variance constrained problem can be transformed into a LMI problem. In [10, 25, 26], the stability and stabilization of the T-S fuzzy system is determined by solving a set of LMIs. It is well known that the LMI problem can be solved numerically using an interior-point method [1].

For practical systems, the states of a system are often not readily available. Under such circumstances, some papers $[11,13,24]$ deal with the fuzzy observer design problem for the T-S fuzzy models. It allows the designers to find a common positive definite matrix, which can be solved by LMI method for the closed-loop systems. This paper focuses on the fuzzy control problem of continuous $\mathrm{T}-\mathrm{S}$ fuzzy stochastic systems under the assumption that the complete state vector cannot be measured. According to the fuzzy observer design concept and fuzzy control theory, an observer-based fuzzy controller design methodology is developed to achieve specified state variance constraints for the T-S fuzzy models. To validate the effectiveness of the proposed observer-based fuzzy controller design method, a numerical example is taken for a T-S fuzzy model subject to the individual state variance constraints. 


\section{OBSERVER-BASED T-S FUZZY SYSTEMS}

Studies on universal approximation of fuzzy systems have achieved great progress in the past few years. In reality, general fuzzy systems are mainly of two classes: Mamdani fuzzy systems [14] and T-S fuzzy systems [19]. The main difference between these two classes lies in their consequent of the fuzzy rules. In this paper, a T-S type fuzzy model is used to construct a nonlinear stochastic system as follows.

\section{Plant Rule i:}

IF $x_{1}(t)$ is $M_{i 1} \ldots$ and $x_{n_{x}}(t)$ is $M_{i n_{x}}$,

THEN $\dot{x}(t)=\boldsymbol{A}_{i} x(x)+\boldsymbol{B}_{i} u(t)+\boldsymbol{D}_{i} v(t)$,

$$
y(t)=C_{i} x(t)+\boldsymbol{E}_{i} \mu(t), i=1,2, \ldots, r,
$$

where $x(t) \in \Re^{n_{x}}$ is the state vector; $u(t) \in \mathfrak{R}^{n_{u}}$ is the control input vector; and $y(t) \in \Re^{n_{y}}$ is the control output vector in $i$-th rule. The $v(t) \in \mathfrak{R}^{n_{v}}$ and $\mu(t) \in \mathfrak{R}^{n_{\mu}}$ are stationary zero-mean mutually independent white noise processes with covariance $\boldsymbol{V}>0$ and $\Omega>0$, respectively. The matrices, $\boldsymbol{A}_{i} \in \mathfrak{R}^{n_{x} \times n_{x}}, \boldsymbol{B}_{i} \in \mathfrak{R}^{n_{x} \times n_{u}}, \boldsymbol{C}_{i} \in \mathfrak{R}^{n_{y} \times n_{x}}, \boldsymbol{D}_{i} \in$ $\Re^{n_{x} \times n_{v}}$, and $\boldsymbol{E}_{i} \in \mathfrak{R}^{n_{y} \times n_{\mu}}$ are constant; $i=1,2, \ldots, r$ and $r$ is the number of IF-THEN rules. The $M_{i j}$ are fuzzy sets and it is assumed that $\boldsymbol{B}_{i}$ is full-column rank. Besides, the pairs $\left(\boldsymbol{A}_{i}, \boldsymbol{B}_{i}\right)$ and $\left(\boldsymbol{A}_{i}, \boldsymbol{C}_{i}\right)$ are controllable and observable, respectively.

The state and output equations for the system can be represented in term of the rules (1) as

$$
\dot{x}(t)=\sum_{i=1}^{r} h_{i}(t) \boldsymbol{A}_{i} x(t)+\sum_{i=1}^{r} h_{i}(t) \boldsymbol{B}_{i} u(t)+\sum_{i=1}^{r} h_{i}(t) \boldsymbol{D}_{i} v(t),
$$

$$
y(t)=\sum_{i=1}^{r} h_{i}(t) \boldsymbol{C}_{i} x(t)+\sum_{i=1}^{r} h_{i}(t) \boldsymbol{E}_{i} \mu(t)
$$

where $h_{i}(t)=\omega_{i}(t) / \sum_{i=1}^{r} \omega_{i}(t), \omega_{i}(t)=\prod_{j=1}^{n_{x}} M_{i j}\left(x_{j}(t)\right)$ and $M_{i j}\left(x_{j}(t)\right)$ is the grade of membership of $x_{j}(t)$ in $M_{i j}(t) ; \omega_{i}$ $(t)$ is the weight of the $i$-th rule.

In some nonlinear systems, the system states usually cannot be completely measured. Therefore, the designers need to design the fuzzy observers to estimate the states for the fuzzy system in order to implement the fuzzy controller. In [4], the authors considered the socalled separation property for a controller and an observer design for the linear stochastic systems. The fuzzy observers require to satisfy the condition $x(t)-$ $\hat{x}(t) \rightarrow 0$ when $t \rightarrow \infty$, where $\hat{x}(t)$ denotes the estimated state vector of the fuzzy observer. In this paper, the fuzzy observer is described as follows:

\section{Observer Rule i:}

$$
\begin{aligned}
& \text { IF } x_{1}(\mathrm{t}) \text { is } M_{i 1} \ldots \text { and } x_{n_{x}}(t) \text { is } M_{i n_{x}}, \\
& \text { THEN } \dot{\hat{x}}(t)=\boldsymbol{A}_{i} \hat{x}(t)+\boldsymbol{B}_{i} u(t)+\boldsymbol{K}_{i}(y(t)-\hat{y}(t)), \\
& \hat{y}(t)=\boldsymbol{C}_{i} \hat{x}(t), i=1,2, \ldots, r,
\end{aligned}
$$

where $\boldsymbol{K}_{i} \in \mathfrak{R}^{n_{x} \times n_{y}}$ are observer gain matrices and $\hat{x}(t) \in \Re^{n_{x}}$ is the state vector of observer. The $y(t)$ and $\hat{y}(t)$ are the output of the fuzzy system and the fuzzy observer, respectively. Then, the final estimated state and output of the fuzzy observer are characterized as follows.

$$
\begin{aligned}
\dot{\hat{x}}(t) & =\sum_{i=1}^{r} h_{i}(t) \boldsymbol{A}_{i} \hat{x}(t)+\sum_{i=1}^{r} h_{i}(t) \boldsymbol{B}_{i} u(t) \\
& +\sum_{i=1}^{r} \sum_{j=1}^{r} h_{i}(t) h_{j}(t) \boldsymbol{K}_{i} \boldsymbol{C}_{j}[x(t)-\hat{x}(t)] \\
& +\sum_{i=1}^{r} \sum_{j=1}^{r} h_{i}(t) h_{j}(t) \boldsymbol{K}_{i} \boldsymbol{E}_{j} \mu(t), \\
\hat{y}(t) & =\sum_{i=1}^{r} h_{i}(t) \boldsymbol{C}_{i} \hat{x}(t),
\end{aligned}
$$

The same weight $h_{i}(t)$ of $i$-th rule of the fuzzy system (2) and (3) is used for the fuzzy observer (5) and (6). The desired parameters of the fuzzy observer are the gain matrices $\boldsymbol{K}_{i}$ in each rule.

In this paper, the concept of PDC $[2,3,7,12,22$, $23,25,26]$ is used to synthesize observer-based fuzzy control laws for the nonlinear system, which is represented by continuous T-S type fuzzy stochastic model (1). The basic idea of the PDC approach is to design the feedback gains for each rule in the fuzzy models. Linear control design techniques can be used to design these linear controllers for each rule. Hence, the nonlinear system controller can be blended by local linear fuzzy controllers sharing the same fuzzy sets with the continuous T-S type fuzzy stochastic model (1). By using the observed states from the fuzzy observer, the observerbased fuzzy controller becomes

\section{Observer-based Fuzzy Controller Rule i:}

IF $x_{1}(t)$ is $M_{i 1} \ldots$ and $x_{n_{x}}(t)$ is $M_{i n_{x}}$

THEN $u(t)=\boldsymbol{G}_{i} \hat{x}(t), \quad i=1,2, \ldots, r$,

where $i=1,2, \ldots, r$ and $r$ is the number of IF-THEN rule. The overall observer-based fuzzy controller becomes

$$
u(t)=\sum_{i=1}^{r} h_{i}(t) \boldsymbol{G}_{i} \hat{x}(t)
$$


By substituting (8) into (2) and (5), state and observer equations of the fuzzy system can be described as follows.

$$
\begin{aligned}
\dot{x}(t) & =\sum_{i=1}^{r} h_{i}(t) \boldsymbol{A}_{i} x(t)+\sum_{i=1}^{r} \sum_{j=1}^{r} h_{i}(t) h_{j}(t) \boldsymbol{B}_{i} \boldsymbol{G}_{j} \hat{x}(t) \\
& +\sum_{i=1}^{r} h_{i}(t) \boldsymbol{D}_{i} v(t) \\
\dot{\hat{x}}= & \sum_{i=1}^{r} \sum_{j=1}^{r} h_{i}(t) h_{j}(t)\left(\boldsymbol{A}_{i}+\boldsymbol{B}_{i} \boldsymbol{G}_{j}\right) \hat{x}(t) \\
& +\sum_{i=1}^{r} \sum_{j=1}^{r} h_{i}(t) h_{j}(t) \boldsymbol{K}_{i} \boldsymbol{C}_{j}[x(t)-\hat{x}(t)] \\
& +\sum_{i=1}^{r} \sum_{j=1}^{r} h_{i}(t) h_{j}(t) \boldsymbol{K}_{i} \boldsymbol{E}_{j} \mu(t) .
\end{aligned}
$$

Let $\tilde{x}(t)=x(t)-\hat{x}(t), \boldsymbol{R}_{i j}=\frac{\left(\boldsymbol{A}_{i}+\boldsymbol{B}_{i} \boldsymbol{G}_{j}\right)+\left(\boldsymbol{A}_{j}+\boldsymbol{B}_{j} \boldsymbol{G}_{i}\right)}{2}$ and $\tilde{\boldsymbol{R}}_{i j}=\frac{\boldsymbol{B}_{i} \boldsymbol{G}_{j}+\boldsymbol{B}_{j} \boldsymbol{G}_{i}}{2}, i<j \leq r$, then (9) can be rewritten as $\dot{x}(t)=\sum_{i=1}^{r} h_{i}(t) h_{j}(t)\left(\boldsymbol{A}_{i}+\boldsymbol{B}_{i} \boldsymbol{G}_{i}\right) x(t)+2 \sum_{i<j} h_{i}(t) h_{j}(t) \boldsymbol{R}_{i j} x(t)$

$$
-\left[\sum_{i=1}^{r} h_{i}(t) h_{j}(t) \boldsymbol{B}_{i} \boldsymbol{G}_{i} \tilde{x}(t)+2 \sum_{i<j}^{r} h_{i}(t) h_{j}(t) \tilde{\boldsymbol{R}}_{i j} \tilde{x}(t)\right]
$$$$
+\sum_{i=1}^{r} h_{i}(t) \boldsymbol{D}_{i} v(t)
$$

The observer error dynamics becomes

$$
\begin{aligned}
\dot{\tilde{x}}(t) & =\sum_{i=1}^{r} h_{i} h_{j}(t)\left(\boldsymbol{A}_{i}-\boldsymbol{K}_{i} \boldsymbol{C}_{i}\right) \tilde{x}(t)+2 \sum_{i<j} h_{i}(t) h_{j}(t) \boldsymbol{H}_{i j} \tilde{x}(t) \\
& -\left[\sum_{i=1}^{r} h_{i}(t) h_{j}(t) \boldsymbol{K}_{i} \boldsymbol{E}_{i} \mu(t)+2 \sum_{i<j}^{r} h_{i}(t) h_{j}(t) \tilde{\boldsymbol{H}}_{i j} \mu(t)\right] \\
& -\sum_{i=1}^{r} h_{i}(t) \boldsymbol{D}_{i} v(t),
\end{aligned}
$$

where $\boldsymbol{H}_{i j}=\frac{\left(\boldsymbol{A}_{i}-\boldsymbol{K}_{i} \boldsymbol{C}_{j}\right)+\left(\boldsymbol{A}_{j}-\boldsymbol{K}_{j} \boldsymbol{C}_{i}\right)}{2}$ and $\tilde{\boldsymbol{H}}_{i j}=$ $\frac{\boldsymbol{K}_{i} \boldsymbol{E}_{j}+\boldsymbol{K}_{j} \boldsymbol{E}_{i}}{2}$. Augmenting (11) and (12) yields:

$$
\begin{aligned}
\dot{\chi}(t) & =\sum_{i=1}^{r} \sum_{k=1}^{r} h_{i}(t) h_{i}(t) h_{k}(t)\left[\boldsymbol{L}_{i} \chi(t)+\boldsymbol{N}_{i k} \bar{v}(t)\right] \\
& +2 \sum_{i<j}^{r} h_{i}(t) h_{j}(t)\left[\boldsymbol{L}_{i j} \chi(t)+\boldsymbol{N}_{i j} \bar{v}(t)\right],
\end{aligned}
$$

where

$$
\begin{aligned}
& \chi(t)=\left[\begin{array}{l}
x(t) \\
\tilde{x}(t)
\end{array}\right], \bar{v}(t)=\left[\begin{array}{l}
v(t) \\
\mu(t)
\end{array}\right], \\
& \boldsymbol{L}_{i}=\left[\begin{array}{cc}
\boldsymbol{A}_{i}+\boldsymbol{B}_{i} \boldsymbol{G}_{i} & -\boldsymbol{B}_{i} \boldsymbol{G}_{i} \\
0 & \boldsymbol{A}_{i}-\boldsymbol{K}_{i} \boldsymbol{C}_{i}
\end{array}\right], \boldsymbol{L}_{i j}=\left[\begin{array}{cc}
\boldsymbol{R}_{i j} & -\tilde{\boldsymbol{R}}_{i j} \\
0 & \boldsymbol{H}_{i j}
\end{array}\right], \\
& \boldsymbol{N}_{i k}=\left[\begin{array}{cc}
\boldsymbol{D}_{k} & 0 \\
\boldsymbol{D}_{k} & -\boldsymbol{K}_{i} \boldsymbol{E}_{i}
\end{array}\right], \boldsymbol{N}_{i j}=\left[\begin{array}{cc}
0 & 0 \\
0 & -\tilde{\boldsymbol{H}}_{i j}
\end{array}\right] .
\end{aligned}
$$

If $\boldsymbol{L}_{i}$ is a stable matrix, the state covariance matrix $\boldsymbol{X}_{i}$ of each subsystem of (13) can be defined by [4, 13, 23, 24]

$$
\boldsymbol{X}_{i}=\lim _{t \rightarrow \infty} E\left[\chi(t) \chi(t)^{T}\right]
$$

Let the common covariance matrix for (13) be $\boldsymbol{X}$ such that

$$
\boldsymbol{X}=\boldsymbol{X}_{i}=\left[\begin{array}{cc}
\boldsymbol{X}_{a a} & \boldsymbol{X}_{a b} \\
\boldsymbol{X}_{a b}^{T} & \boldsymbol{X}_{b b}
\end{array}\right], i=1,2, \ldots, r
$$

and $\boldsymbol{X}=\boldsymbol{X}^{T}>0$, then $\boldsymbol{X}$ satisfies the following Lyapunov equation for each rule [4, 13, 23-24]:

$$
\boldsymbol{L}_{i} \boldsymbol{X}+\boldsymbol{X} \boldsymbol{L}_{i}^{T}+\boldsymbol{N}_{i} \boldsymbol{\Phi} \boldsymbol{N}_{i}^{T}=0
$$

where $\boldsymbol{\Phi}=\left[\begin{array}{ll}\boldsymbol{V} & 0 \\ 0 & \boldsymbol{\Omega}\end{array}\right]$

A T-S type fuzzy observer and an observer-based fuzzy controller are used to construct a nonlinear stochastic system as above. Based on the common state covariance matrix defined in (15), references [15-17] provided the conditions and solutions for the optimal filter gains $\boldsymbol{K}_{i}$ as follows:

$$
\begin{aligned}
& \boldsymbol{K}_{i}=\boldsymbol{X}_{b b} \boldsymbol{C}_{i}^{T}\left(\boldsymbol{E}_{i} \Omega \boldsymbol{E}_{i}^{T}\right)^{-1} \\
& \left(\boldsymbol{A}_{i}+\boldsymbol{B}_{i} \boldsymbol{G}_{i}\right) \boldsymbol{X}_{a a}+\boldsymbol{X}_{a a}\left(\mathrm{~A}_{i}+\boldsymbol{B}_{i} \boldsymbol{G}_{i}\right)^{T}-\boldsymbol{B}_{i} \boldsymbol{G}_{i} \boldsymbol{X}_{b b} \\
& -\boldsymbol{X}_{b b}\left(\boldsymbol{B}_{i} \boldsymbol{G}_{i}\right)^{T}+\boldsymbol{D}_{i} \boldsymbol{V} \boldsymbol{D}_{i}^{T}=0 \\
& \boldsymbol{A}_{i} \boldsymbol{X}_{b b}+\boldsymbol{X}_{b b}\left(\boldsymbol{A}_{i}-\boldsymbol{K}_{i} \boldsymbol{C}_{i}\right)^{T}+\boldsymbol{D}_{i} \boldsymbol{V} \boldsymbol{D}_{i}^{T}=0
\end{aligned}
$$

where $\boldsymbol{X}_{a \boldsymbol{a}}>0, \boldsymbol{X}_{\boldsymbol{b} b}>0, \boldsymbol{X}_{\boldsymbol{a} b}=\boldsymbol{X}_{\boldsymbol{b} \boldsymbol{b}}$ and $\left(\boldsymbol{X}_{\boldsymbol{a} a}-\boldsymbol{X}_{\boldsymbol{b} \boldsymbol{b}}\right)>0$ are defined in (15). Note that the above conditions are necessary and sufficient [17] for the existence of optimal estimators since they satisfy the Wiener-Hopf equation [15]. From the results of [16-17], it can be found that the optimal filter gain $\boldsymbol{K}_{i}=\boldsymbol{X}_{b b} \boldsymbol{C}_{i}^{T}\left(\boldsymbol{E}_{i} \Omega \boldsymbol{E}_{i}^{T}\right)^{-1}$ leads to the fact that the steady state error between the system state $x(t)$ and the estimated state $\hat{x}(t)$ converges to zero 
when $t \rightarrow \infty$. Note that the state covariance matrix $\boldsymbol{X}$ defined in (15) satisfies the following Lyapunov equation:

$$
\begin{aligned}
& \left(\boldsymbol{A}_{i}+\boldsymbol{B}_{i} \boldsymbol{G}_{i}\right)\left(\boldsymbol{X}_{a a}-\boldsymbol{X}_{b b}\right)+\left(\boldsymbol{X}_{a a}-\boldsymbol{X}_{b b}\right)\left(\boldsymbol{A}_{i}+\boldsymbol{B}_{i} \boldsymbol{G}_{i}\right)^{T} \\
& +\boldsymbol{X}_{b b} \boldsymbol{C}_{i}^{T}\left(\boldsymbol{E}_{i} \Omega \boldsymbol{E}_{i}^{T}\right)^{-1} \boldsymbol{C}_{i} \boldsymbol{X}_{b b}=0
\end{aligned}
$$

Besides, the assumption $\boldsymbol{X}_{a b}=\boldsymbol{X}_{b b}$ implies that the estimate $\hat{x}$ and the error $\tilde{x}$ are orthogonal, i.e., $E\left[\hat{x} \tilde{x}^{T}\right]$ $=0$. Without loss of generality, this assumption has been applied in the design of optimal filter for the continuous-time systems [16, 18]. From (17), if continuous T-S type fuzzy model (1) is corrupted only by state noise without measurement noise (i.e., $\Omega=0$ ), then the optimal filter gain $\boldsymbol{K}_{i}$ does not exist. In next section, the observer-based fuzzy control problem is solved such that the state variance constraints are satisfied.

\section{OBSERVER-BASED FUZZY CONTROL WITH STATE VARIANCE CONSTRAINTS}

In this section, the Lyapunov approach is used to discuss and analyze the stability conditions for the T-S fuzzy models. As well as, the individual state variance constraints are also considered in the observer-based fuzzy controller design process. A variance constrained fuzzy control methodology for continuous T-S fuzzy stochastic systems has been developed in [6]. However, it did not consider the observed-state feedback control technique. To offer a lucid presentation of the observerbased fuzzy control theory for continuous T-S fuzzy model (1), we consider the stability analysis for the TS fuzzy model (1) by applying the fuzzy observers. The stability conditions of the observer-based fuzzy control problem are stated by using the optimal estimations defined in (17-19) via the following theorem.

\section{Theorem 1}

For a T-S fuzzy model (1), which is driven by (8) and (10), the closed-loop system is possessed by an optimal filter gain $\boldsymbol{K}_{i}=\boldsymbol{X}_{b b} \boldsymbol{C}_{i}^{T}\left(\boldsymbol{E}_{i} \boldsymbol{\Omega} \boldsymbol{E}_{i}^{T}\right)^{-1}$ with $\boldsymbol{X}_{a a}>0, \boldsymbol{X}_{b b}$ $>0$ and $\boldsymbol{X}_{a b}=\boldsymbol{X}_{b b}$, which satisfy equations (17-19). If there exist common positive definite matrices $\bar{X}_{a a}$ and $\overline{\boldsymbol{X}}_{a a}-\boldsymbol{X}_{b b}>0$ satisfying the following conditions

$$
\boldsymbol{A}_{i} \boldsymbol{X}_{b b}+\boldsymbol{X}_{b b} \boldsymbol{A}_{i}^{T}-\boldsymbol{X}_{b b} \boldsymbol{C}_{i}^{T}\left(\boldsymbol{E}_{i} \Omega \boldsymbol{E}_{i}^{T}\right)^{-1} \boldsymbol{C}_{i} \boldsymbol{X}_{b b}+\boldsymbol{D}_{i} \boldsymbol{V D} \boldsymbol{D}_{i}^{T}=0
$$

$$
\begin{aligned}
& \left(\boldsymbol{A}_{i}+\boldsymbol{B}_{i} \boldsymbol{G}_{i}\right)\left(\overline{\boldsymbol{X}}_{a a}-\boldsymbol{X}_{b b}\right)+\left(\overline{\boldsymbol{X}}_{a a}-\boldsymbol{X}_{b b}\right)\left(\boldsymbol{A}_{i}+\boldsymbol{B}_{i} \boldsymbol{G}_{i}\right)^{T} \\
& +\boldsymbol{X}_{b b} \boldsymbol{C}_{i}^{T}\left(\boldsymbol{E}_{i} \boldsymbol{\Omega} \boldsymbol{E}_{i}^{T}\right)^{-1} \boldsymbol{C}_{1} \boldsymbol{X}_{b b}<0
\end{aligned}
$$

$$
\boldsymbol{R}_{i j}\left(\overline{\boldsymbol{X}}_{a a}-\boldsymbol{X}_{b b}\right)+\left(\overline{\boldsymbol{X}}_{a a}-\boldsymbol{X}_{b b}\right) \boldsymbol{R}_{i j}^{T}<0, i<j \leq r
$$

then the equilibrium of the closed-loop continuous T-S fuzzy stochastic control system (11) is asymptotically stable in the large and $\overline{\boldsymbol{X}}_{a a}>\boldsymbol{X}_{a a}$.

\section{Proof:}

From the statements of above section, it is clear that the matrix $\boldsymbol{K}_{i}$ performs an optimal filter gain if and only if there exist matrices $\boldsymbol{X}_{a a}>0$ and $\boldsymbol{X}_{b b}>0$ such that (17-19) are all satisfied with $\boldsymbol{X}_{a b}=\boldsymbol{X}_{b b}$ defined in (15). Substituting (17) into (19) and rearranging yields

$$
\boldsymbol{A}_{i} \boldsymbol{X}_{b b}+\boldsymbol{X}_{b b} \boldsymbol{A}_{i}^{T}+\boldsymbol{D}_{i} \boldsymbol{V D} \boldsymbol{D}_{i}^{T}=\boldsymbol{X}_{b b} \boldsymbol{C}_{i}^{T}\left(\boldsymbol{E}_{i} \Omega \boldsymbol{E}_{i}^{T}\right)^{-1} \boldsymbol{C}_{i} \boldsymbol{X}_{b b},
$$

which is equivalent to (21). Subtracting (20) from (22), one has

$$
\left(\boldsymbol{A}_{i}+\boldsymbol{B}_{i} \boldsymbol{G}_{i}\right)\left(\overline{\boldsymbol{X}}_{a a}-\boldsymbol{X}_{a a}\right)+\left(\overline{\boldsymbol{X}}_{a a}-\boldsymbol{X}_{a a}\right)\left(\boldsymbol{A}_{i}+\boldsymbol{B}_{i} \boldsymbol{G}_{i}\right)^{T}<0
$$

Using the similar method yields

$$
\boldsymbol{R}_{i j}\left(\overline{\boldsymbol{X}}_{a a}-\boldsymbol{X}_{a a}\right)+\left(\overline{\boldsymbol{X}}_{a a}-\boldsymbol{X}_{a a}\right) \boldsymbol{R}_{i j}^{T}<0
$$

It is easy to find that the optimal filter gain $\boldsymbol{K}_{i}$ defined in (17-19) leads to the fact $\tilde{x}(t) \rightarrow 0$ when $t \rightarrow \infty$. From Theorem 3 of [21], one can deduce that if there exist a common positive definite error state covariance matrix $\left(\overline{\boldsymbol{X}}_{a a}-\boldsymbol{X}_{b b}\right)>0$ satisfying (22) and (23), then the equilibrium of continuous fuzzy control system (11) is asymptotically stable in the large due to $\boldsymbol{X}_{b b} \boldsymbol{C}_{i}^{T}$ $\left(\boldsymbol{E}_{i} \Omega \boldsymbol{E}_{i}^{T}\right)^{-1} \boldsymbol{C}_{i} \boldsymbol{X}_{b b} \geq 0$ and $\tilde{x}(t) \rightarrow 0$. In the case of the matrices $\left(\boldsymbol{A}_{i}+\boldsymbol{B}_{i} \boldsymbol{G}_{i}\right)$ and $\boldsymbol{R}_{i j}$ being stable, one can obtain that $\overline{\boldsymbol{X}}_{a a}-\boldsymbol{X}_{a a}>0$ from (25) and (26). Hence, it can be concluded that if conditions (21-23) are all satisfied with $\overline{\boldsymbol{X}}_{a a}-\overline{\boldsymbol{X}}_{a a}^{T}>0$ and $\left(\overline{\boldsymbol{X}}_{a a}-\boldsymbol{X}_{b b}\right)>0$, then the equilibrium of the closed-loop nominal continuous T-S fuzzy stochastic control system (11) is asymptotically stable in the large and $\overline{\boldsymbol{X}}_{a a}$ is the upper bound of matrix $\boldsymbol{X}_{a a}$, i.e., $\overline{\boldsymbol{X}}_{a a}>\boldsymbol{X}_{a a}$.

Applying the results of Theorem 1, the common state covariance matrix $\boldsymbol{X}$ has an upper bound matrix. It can be defined as $\overline{\boldsymbol{X}}$, i.e.,

$$
\overline{\boldsymbol{X}}=\left[\begin{array}{ll}
\overline{\boldsymbol{X}}_{a a} & \boldsymbol{X}_{a b} \\
\boldsymbol{X}_{a b}^{T} & \boldsymbol{X}_{b b}
\end{array}\right], i=1,2, \ldots, r
$$


where $\overline{\boldsymbol{X}}$ is the upper bound of common state covariance matrix $\boldsymbol{X}$, i.e., $\overline{\boldsymbol{X}}>\boldsymbol{X}$.

From the stability conditions of Theorem 1, one can obtain LMI stability conditions with respect to $\boldsymbol{Z}_{i}=\boldsymbol{G}_{i}\left(\overline{\boldsymbol{X}}_{a a}-\boldsymbol{X}_{b b}\right)$, and they can be respected in the following theorem.

\section{Theorem 2}

For a T-S fuzzy model (1), which is driven by (8) and (10), the closed-loop system is possessed by an optimal filter gain $\boldsymbol{K}_{i}=\boldsymbol{X}_{b b} \boldsymbol{C}_{i}^{T}\left(\boldsymbol{E}_{i} \Omega \boldsymbol{E}_{i}^{T}\right)^{-1}$ with $\boldsymbol{X}_{a a}>0, \boldsymbol{X}_{b b}$ $>0$ and $\boldsymbol{X}_{a b}=\boldsymbol{X}_{b b}$, which satisfy equations (17-19). If there exist common positive definite matrices $\overline{\boldsymbol{X}}_{a a}$ and $\overline{\boldsymbol{X}}_{a a}-\boldsymbol{X}_{b b}>0$, satisfying the following conditions, then the stability conditions (22-23) of Theorem 1 are achieved.

$$
\begin{aligned}
& {\left[\begin{array}{cc}
\boldsymbol{\Theta}_{i} & \boldsymbol{X}_{b b} \boldsymbol{C}_{i}^{T} \\
\boldsymbol{C}_{i} \boldsymbol{X}_{b b} & -\left(\boldsymbol{E}_{i} \boldsymbol{\Omega} \boldsymbol{E}_{i}^{T}\right)
\end{array}\right]<0, i=1,2, \ldots, r,} \\
& \boldsymbol{A}_{i} \overline{\boldsymbol{X}}_{a a}+\overline{\boldsymbol{X}}_{a a} \boldsymbol{A}_{i}^{T}-\boldsymbol{A}_{i} \boldsymbol{X}_{b b}-\boldsymbol{X}_{b b} \boldsymbol{A}_{i}^{T}+\boldsymbol{A}_{j} \overline{\boldsymbol{X}}_{a a} \\
& +\overline{\boldsymbol{X}}_{a a} \boldsymbol{A}_{j}^{T}-\boldsymbol{A}_{j} \boldsymbol{X}_{b b}-\boldsymbol{X}_{b b} \boldsymbol{A}_{j}^{T}+\boldsymbol{B}_{i} \boldsymbol{Z}_{j}+\boldsymbol{B}_{j} \boldsymbol{Z}_{i} \\
& +\left(\boldsymbol{B}_{i}^{T} \boldsymbol{Z}_{j}^{T}+\boldsymbol{B}_{j}^{T} \boldsymbol{Z}_{i}^{T}\right)<0, i<j
\end{aligned}
$$

where $\boldsymbol{Z}_{i}=\boldsymbol{G}_{i}\left(\overline{\boldsymbol{X}}_{a a}-\boldsymbol{X}_{b b}\right)$ and $\Theta_{i}$ are defined as

$$
\begin{aligned}
\boldsymbol{\Theta}_{i} & =\boldsymbol{A}_{i} \overline{\boldsymbol{X}}_{a a}+\overline{\boldsymbol{X}}_{a a} \boldsymbol{A}_{i}^{T}-\boldsymbol{A}_{i} \boldsymbol{X}_{b b}-\boldsymbol{X}_{b b} \boldsymbol{A}_{i}^{T} \\
& +\boldsymbol{B}_{i} \boldsymbol{Z}_{i}+\boldsymbol{Z}_{i}^{T} \boldsymbol{B}_{i}^{T}
\end{aligned}
$$

The proof of Theorem 2 can be obtained via Schur complement [1]. By Schur complement, one can get that (22) and (23) are equivalent to (28) and (29). In Theorem 2, the stability conditions of Theorem 1 are transformed into LMIs. Based on the stability conditions of Theorem 2, this paper not only consider to design stable observer-based fuzzy controllers but also to achieve individual state variance constraints. The state variance performance constrained design problem is an application of upper bound state covariance control approach $[5,22,23]$. The purpose of this paper is to find the set of controllers $\boldsymbol{G}_{i}$, which satisfy the stability conditions of Theorem 1 , such that the upper bound state covariance matrix $\overline{\boldsymbol{X}}_{a a}$ satisfies the following variance performance objectives:

$$
\begin{aligned}
& \lim _{t \rightarrow \infty} E\left[x_{\varphi}^{2}(t)\right]=\left[\boldsymbol{X}_{a a}\right]_{\varphi \varphi} \leq\left[\overline{\boldsymbol{X}}_{a a}\right]_{\varphi \varphi} \leq \sigma_{\varphi}^{2}, \\
& \varphi=1,2, \ldots, n_{x},
\end{aligned}
$$

where $\sigma_{\varphi}$ denote the Root-Mean-Squared (RMS) constraint for the variances of system states. This problem is referred to as the state variance constrained design using the observer-based fuzzy control. The purpose of this paper is to find common positive definite matrices $\overline{\boldsymbol{X}}_{a a}$ and $\left(\overline{\boldsymbol{X}}_{a a}-\boldsymbol{X}_{b b}\right)$ to satisfy stability conditions (21-23) subject to the state variance constraint (31). For this purpose, this paper provides a formula to find the observer-based fuzzy controllers for achieving individual state variance constraint (31).

\section{Theorem 3}

If stability conditions (21), (28-29) and the following LMIs are satisfied for a given $\sigma_{\varphi}>0$. Then, the TS fuzzy model (1), driven by (8) and (10), is stable and the state variance constraint (31) is achieved.

$$
\left[\begin{array}{cc}
\sigma_{\varphi}^{2} & \boldsymbol{I}_{\varphi} \overline{\boldsymbol{X}}_{a a} \\
\overline{\boldsymbol{X}}_{a a} \boldsymbol{I}_{\varphi}^{T} & \overline{\boldsymbol{X}}_{a a}
\end{array}\right]>0, \varphi=1,2, \ldots, n_{x}
$$

where $\boldsymbol{I}_{\varphi}=\left[\begin{array}{lllll}0 & \ldots & 1 & \ldots & 0\end{array}\right] \in \mathfrak{R}^{1 \times n_{x}}$ denotes a row vector with the $\varphi$ th element is 1 and others are 0 .

\section{Proof:}

In order to transform the present control problem into a LMI problem, it is necessary to transform the state variance constrain (31) into a LMI form. Hence, the LMI condition (32) is directly constructed from the state variance constraint (31).

It is noted that the observer-based fuzzy control problem with state variance constraints can be dealt with by the LMI criterions of Theorem 3. These LMI criterions can be solved numerically using an interiorpoint method [1]. In next section, a numerical example is provided to verify the usefulness of the proposed observer-based fuzzy controller design method.

\section{A NUMERICAL EXAMPLE}

To illustrate the proposed observer-based fuzzy control approach, consider a two-rule $(r=2) \mathrm{T}-\mathrm{S}$ fuzzy model, which is described as follows.

\section{Plant Rule 1:}

IF $x_{1}(t)$ is $M_{11}$

$\begin{aligned} \operatorname{THEN} \dot{x}(t) & =\boldsymbol{A}_{1} x(t)+\boldsymbol{B}_{1} u(t)+\boldsymbol{D}_{1} v(t) \\ y(t) & =\boldsymbol{C}_{1} x(t)+\boldsymbol{E}_{1} u(t)\end{aligned}$

Plant Rule 2:

IF $x_{1}(\mathrm{t})$ is $M_{2}$

$$
\text { THEN } \dot{x}(t)=\boldsymbol{A}_{2} x(t)+\boldsymbol{B}_{2} u(t)+\boldsymbol{D}_{2} v(t)
$$$$
y(t)=\boldsymbol{C}_{2} x(t)+\boldsymbol{E}_{2} \mu(t)
$$ 
where

$$
\begin{aligned}
& \boldsymbol{A}_{1}=\left[\begin{array}{ccc}
0 & 0 & 2.5 \\
1 & -1 & 2 \\
0 & -1.25 & 0
\end{array}\right], \boldsymbol{A}_{2}=\left[\begin{array}{ccc}
0.41 & -0.9 & 3.75 \\
1 & -1 & 2 \\
0 & -1.25 & 0
\end{array}\right], \\
& \boldsymbol{B}_{1}=\left[\begin{array}{c}
0 \\
1.5 \\
0
\end{array}\right], \boldsymbol{B}_{2}=\left[\begin{array}{c}
0 \\
0.6 \\
0
\end{array}\right], \boldsymbol{C}_{1}=\boldsymbol{C}_{2}=\left[\begin{array}{lll}
5 & 2 & 1
\end{array}\right], \\
& \boldsymbol{D}_{1}=\left[\begin{array}{c}
0.05 \\
0 \\
0.05
\end{array}\right], \boldsymbol{D}_{2}=\left[\begin{array}{c}
0 \\
0 \\
0.05
\end{array}\right], \boldsymbol{E}_{1}=\boldsymbol{E}_{2}=1,
\end{aligned}
$$

In Figure 1, the fuzzy sets for Plant Rule 1 and 2 are described by two triangular membership functions. In this example, it is assumed that the state variance constraints of this system have the following forms:

$$
\left[\boldsymbol{X}_{a a}\right]_{11} \leq 6,\left[\boldsymbol{X}_{a a}\right]_{22} \leq 3,\left[\boldsymbol{X}_{a a}\right]_{33} \leq 5
$$

Besides, the covariance matrices of zero-mean white noises $v(t)$ and $\mu(t)$ are $\boldsymbol{V}=10$ and $\Omega=1$, respectively. Before finding the solutions of common positive definite upper bound covariance matrix $\overline{\boldsymbol{X}}_{a a}$, the positive definite matrix $\boldsymbol{X}_{b b}$ can be obtained by solving the algebraic Riccati-like Eq. (21).

$$
\boldsymbol{X}_{b b}=\left[\begin{array}{lll}
0.049 & 0.030 & 0.016 \\
0.030 & 0.021 & 0.005 \\
0.016 & 0.005 & 0.019
\end{array}\right]
$$

To carry on, using LMI-toolbox of MATLAB, one can find the solutions of common positive definite upper bound covariance matrix $\overline{\boldsymbol{X}}_{a a}$ and feedback gains $\boldsymbol{G}_{i}$ from LMIs (28-29) and (32).

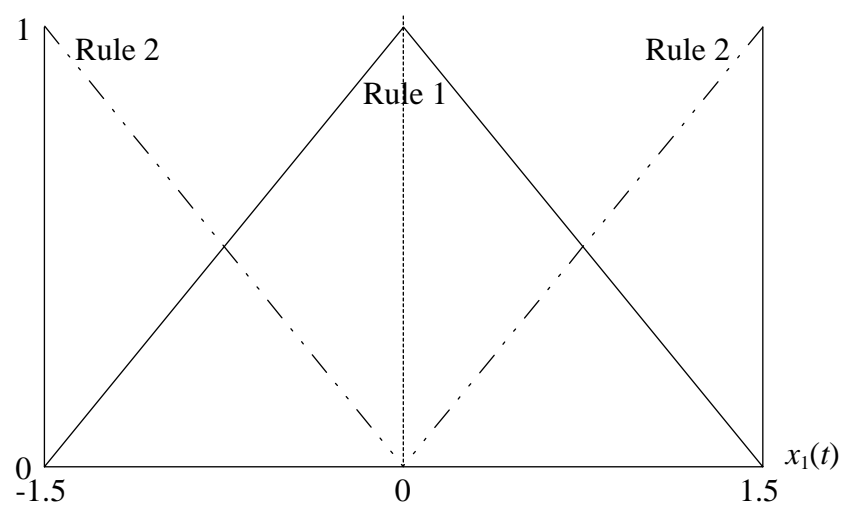

Fig. 1. The membership function of $x_{1}(t)$.

$$
\begin{aligned}
& \overline{\boldsymbol{X}}_{a a}=\left[\begin{array}{ccc}
4.494 & 0.383 & -1.489 \\
0.383 & 2.694 & 0.966 \\
-1.489 & 0.966 & 1.287
\end{array}\right] \\
& \boldsymbol{G}_{1}=\left[\begin{array}{lll}
2.326 & -4.583 & 7.115
\end{array}\right], \\
& \boldsymbol{G}_{2}=\left[\begin{array}{lll}
3.834 & -6.759 & 11.538
\end{array}\right]
\end{aligned}
$$

Applying $\overline{\boldsymbol{X}}_{a a}$ and $\boldsymbol{X}_{b b}$ to construct $\overline{\boldsymbol{X}}$, which is defined in (27), one has

$$
\overline{\boldsymbol{X}}=\left[\begin{array}{cccccc}
4.494 & 0.383 & -1.489 & 0.049 & 0.030 & 0.016 \\
0.383 & 2.694 & 0.966 & 0.030 & 0.021 & 0.005 \\
-1.489 & 0.966 & 1.287 & 0.016 & 0.005 & 0.019 \\
0.049 & 0.030 & 0.016 & 0.049 & 0.030 & 0.016 \\
0.030 & 0.021 & 0.005 & 0.030 & 0.021 & 0.005 \\
0.016 & 0.005 & 0.019 & 0.016 & 0.005 & 0.019
\end{array}\right]
$$

Subtracting $\boldsymbol{X}_{b b}$ from $\overline{\boldsymbol{X}}_{a a}$, one can obtain

$$
\overline{\boldsymbol{X}}_{a a}-X_{b b}=\left[\begin{array}{ccc}
4.445 & 0.353 & -1.504 \\
0.353 & 2.673 & 0.960 \\
-1.504 & 0.960 & 1.268
\end{array}\right]>0
$$

Substituting $\boldsymbol{X}_{b b}$ into (17), the fuzzy observer gains $\boldsymbol{K}_{i}$ can be obtained as follows:

$$
\begin{aligned}
& \boldsymbol{K}_{1}=\left[\begin{array}{lll}
0.321 & 0.198 & 0.107
\end{array}\right]^{T}, \\
& \boldsymbol{K}_{2}=\left[\begin{array}{lll}
0.321 & 0.198 & 0.107
\end{array}\right]^{T}
\end{aligned}
$$

Since conditions (21-23) of Theorem 1 are all satisfied, one can conclude that the closed-loop continuous observer-based T-S fuzzy stochastic control system (11) is asymptotically stable by applying the observer gains (40) and the fuzzy control gains (37). Besides, it also achieved the goal of the state variance performance constraints (34). In the simulation, the initial states are given as $\left[\begin{array}{lll}x_{1}(0) & x_{2}(0) & x_{3}(0)\end{array}\right]^{T}=\left[\begin{array}{ll}0.25 \\ 0.25\end{array}\right.$ $\left.\begin{array}{ll}-0.5 & 0.75\end{array}\right]^{T}$. Figure 2 and Figure 3 show the input signals of $v(t)$ and $\mu(t)$ for the simulations. Applying fuzzy control gains $\boldsymbol{G}_{1}$ and $\boldsymbol{G}_{2}$ in the simulation, Figure 4 shows the responses of control input. In addition, Figure 5, Figure 6 and Figure 7 show the responses of true states $X(t)$ and the estimated state $\hat{X}(t)$ for the controlled T-S fuzzy model (33), respectively. From these simulation results, the state variances of closedloop nonlinear system (33) are calculated as follows: 


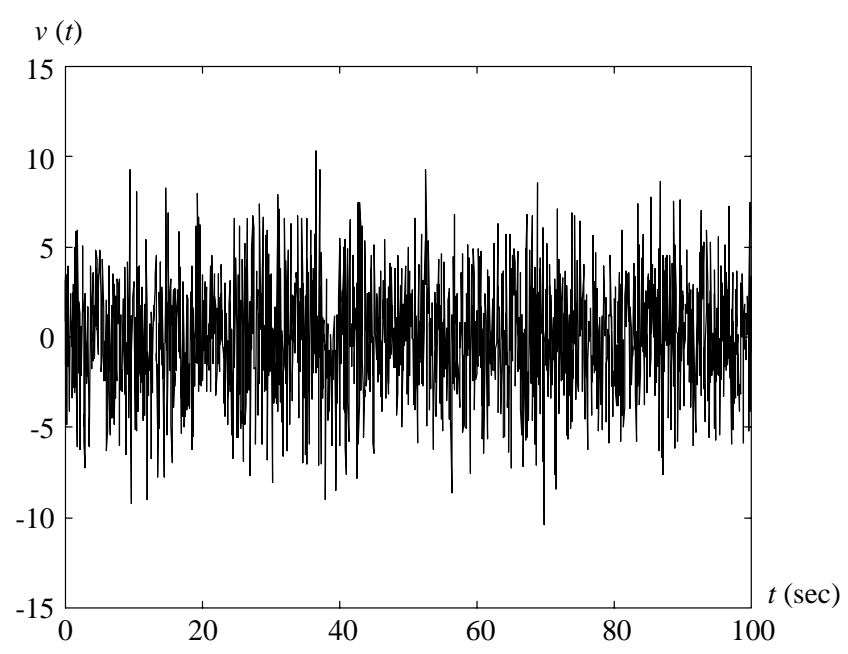

Fig. 2. The input signals of $v(t)$.

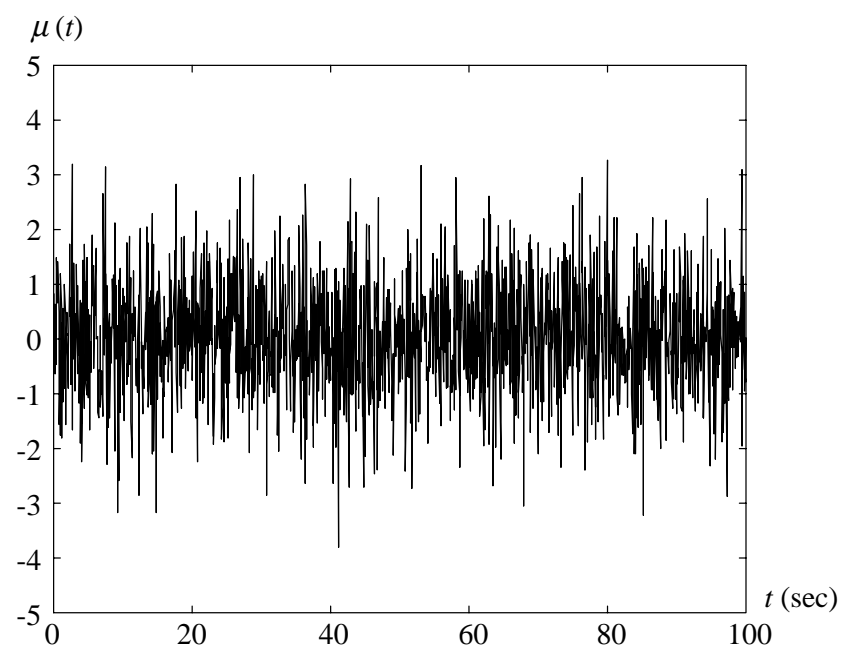

Fig. 3. The input signals of $\mu(t)$.

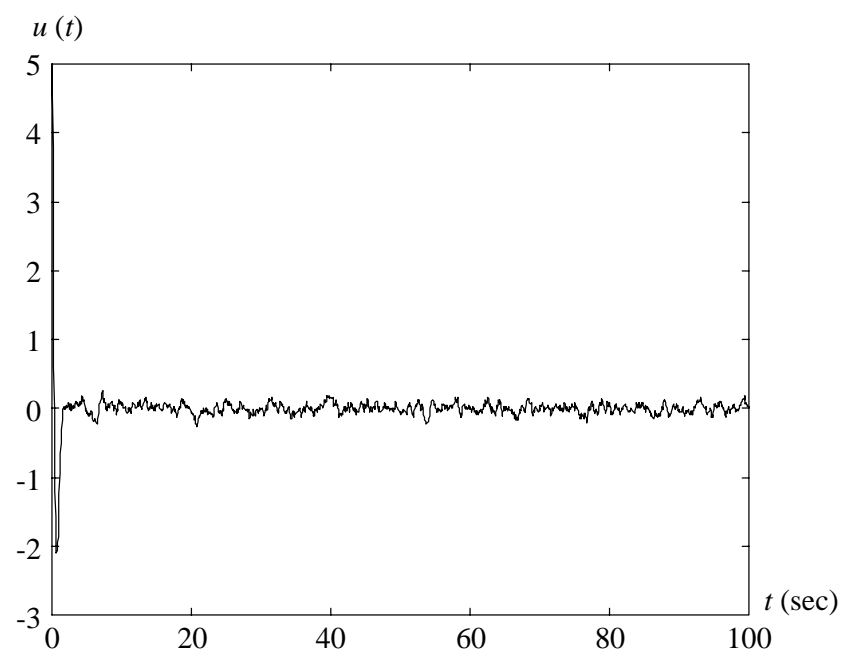

Fig. 4. The responses of control input $u(t)$.

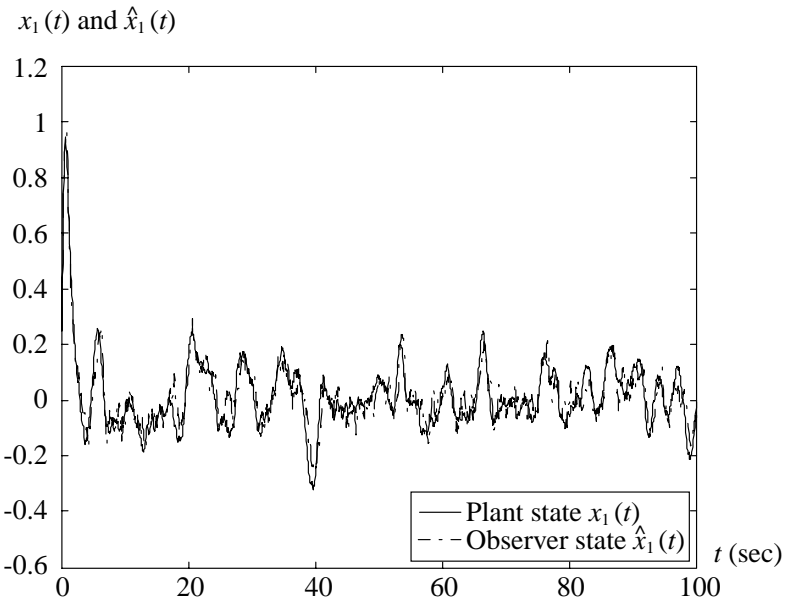

Fig. 5. The responses of true state $x_{1}(t)$ and estimated state $\hat{x}_{1}(t)$ for controlled fuzzy model (33).

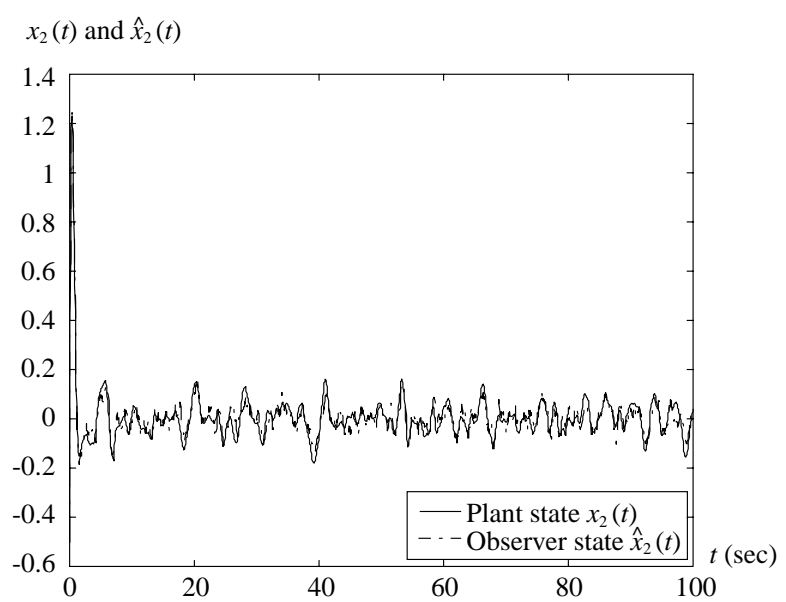

Fig. 6. The responses of true state $x_{2}(t)$ and estimated state $\hat{x}_{2}(t)$ for controlled fuzzy model (33).

$x_{3}(t)$ and $\hat{x}_{3}(t)$

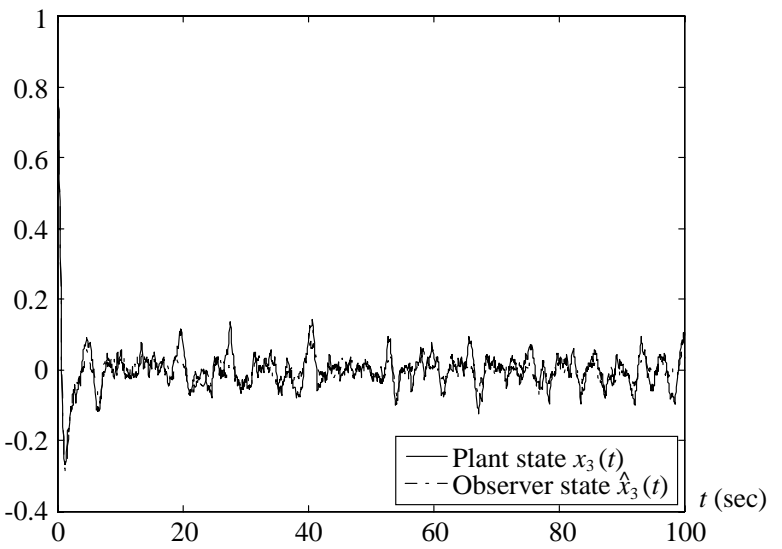

Fig. 7 . The responses of true state $x_{3}(t)$ and estimated state $\hat{x}_{3}(t)$ for controlled fuzzy model (33). 


$$
\begin{aligned}
& \operatorname{var}\left(x_{1}(t)\right)=0.0175, \operatorname{var}\left(x_{2}(t)\right)=0.0124 \\
& \text { and } \operatorname{var}\left(x_{3}(t)\right)=0.0058
\end{aligned}
$$

where $\operatorname{var}\left(x_{\ell}(t)\right)$ denotes the variance of system state $x_{\ell}(t), \ell=1,2$ and 3 . It can be found that the closed-loop fuzzy system is stable and the variance constraints (34) are satisfied.

\section{CONCLUSIONS}

The observer-based fuzzy controller design problem has been solved in this paper subject to individual state variance constraints. The filtering optimal control technique was used to design the observers for the continuous T-S fuzzy stochastic models. The LMI conditions were developed for the existence of observer-based fuzzy controllers, which allows designers to assign a specified upper bound common state covariance matrix to the closed-loop continuous T-S fuzzy models. The PDC concept was applied to design fuzzy controllers for each rule and to solve the solutions of the observer-based fuzzy controllers. The proposed approach aimed at finding observer-based fuzzy controllers such that the closed-loop system is asymptotically stable and the state variance constraints are achieved, simultaneously.

\section{ACKNOWLEDGEMENTS}

This work was supported by the National Science Council of the Republic of China under contract NSC932218-E-019-002.

\section{REFERENCES}

1. Boyd, S., Ghaoui, L.E., Feron, E., and Balakrishnan, V., Linear Matrix Inequalities in Systems and Control Theory, SIAM, Philadelphia, PA (1994).

2. Chang, W.J., "Model-Based Fuzzy Controller Design with Common Observability Gramian Assignment," Journal of Dynamic Systems Measurement and ControlASME, Vol. 123, No. 1, pp. 113-116 (2001).

3. Chang, W.J., "Fuzzy Controller Design via the Inverse Solution of Lyapunov Equations," Journal of Dynamic Systems Measurement and Control-ASME, Vol. 125, No. 1, pp. 42-47 (2003).

4. Chang, W.J. and Chung, H.Y., "A Study of Norm and Variance Constrained Design Using Dynamic Output Feedback for Linear Discrete Systems," International Journal of Control, Vol. 57, No. 2, pp. 473-483 (1993).

5. Chang, W.J. and Sun, C.C., "Constrained Fuzzy Controller Design of Discrete Takagi-Sugeno Fuzzy Models," Fuzzy Sets and Systems, Vol. 133, No. 1, pp.
37-55 (2003).

6. Chang, W.J. and Wu, S.M., "State Variance Constrained Fuzzy Controller Design for Nonlinear TORA systems with Minimizing Control Input Energy," Proceedings of IEEE International Conference on Robot and Automation, Taipei, Taiwan, pp. 2616-2621 (2003).

7. Chung, H.Y. and Chang, W.J., "Constrained Variance Design for Bilinear Stochastic Continuous Systems," IEE Proceedings-Part D: Control Theory and Applications, Vol. 138, No. 2, pp. 145-150 (1991).

8. Chung, H.Y. and Chang, W.J., "Covariance Control with Variance Constraints for Continuous Perturbed Stochastic Systems," Systems \& Control Letters, Vol. 19, No. 5, pp. 413-417 (1992).

9. Collins, Jr. E.G. and Skelton, R.E., "Constrained Variance Design Using State Covariance Assignment," Proceedings of American Control Conference, Seattle, WA, pp. 51-56 (1986).

10. Hong, S.K. and Langari, R., "Robust Fuzzy Control of a Magnetic Bearing System Subject to Harmonic Disturbances," IEEE Transactions on Control Systems Technology, Vol. 8, No. 2, pp. 366 -371 (2000).

11. Jun, Y., Masahiro, N., Hitoshi, K., and Ichikawa, A., "Design of Output Feedback Controllers for TakagiSugeno Fuzzy Systems," Fuzzy Sets and Systems, Vol. 121, No. 1, pp. 127-148 (2001).

12. Kwakernaad, H. and Sivan, R., Linear Optimal Control Systems, John Wiley and Sons, New York (1972).

13. Li, N., Li, S.Y., and Xi, Y.G., "Stability Analysis of TS Fuzzy System Based on Observers," International Journal of Fuzzy Systems, Vol. 5, No. 1, pp. 22-30 (2003).

14. Mamdani, E.H., "Applications of Fuzzy Algorithms for Control of Simple Dynamic Plant," Proceedings of IEEE Institution of Electrical Engineering and Control Science, Vol. 121, No. 12, pp. 1585-1588 (1974).

15. Maybeck, P.S., Stochastic Models, Estimation, and Control, Academic Press, New York (1979).

16. Phillis, Y.A., "Controller Design of Systems with Multiplicative Noise," IEEE Transactions on Automatic Control, Vol. 30, No. 10, pp. 1017-1019 (1985).

17. Phillis, Y.A., "A Smoothing Algorithm for Systems with Multiplicative Noise," IEEE Transactions on Automatic Control, Vol. 33, No. 4, pp. 401-403 (1988).

18. Phillis, Y.A., "Estimation and Control of Systems with Unknown Covariance and Multiplicative Noise," IEEE Transactions on Automatic Control, Vol. 34, No. 10, pp. 1075-1078 (1989).

19. Takagi, T. and Sugeno, M., "Fuzzy Identification of Systems and Its Applications to Modeling and Control," IEEE Transactions on Systems Man and Cybernetics, Vol. 15, No. 1, pp. 116-132 (1985).

20. Tanaka, K., Ikeda, T., and Wang, H.O., “A Unified Approach to Controlling Chaos via an LMI-Based Fuzzy 
Control System Design," IEEE Transactions on Circuits and Systems I: Fundamental Theory and Applications, Vol. 45, No. 10, pp. 1021-1040 (1998).

21. Tanaka, K. and Wang, H.O., Fuzzy Control System Design and Analysis - A Linear Matrix Inequality Approach, John Wiley and Sons, New York (2001).

22. Toivonen, H.T., "Minimum Variance Control of FirstOrder Systems with a Constraint on the Input Amplitude," IEEE Transactions on Automatic Control, Vol. 26, No. 2, pp. 556-558 (1981).

23. Toivonen, H.T., "Variance Constrained Self-Turning Control," Automatica, Vol. 19, No. 4, pp. 415-418 (1983).
24. Tong, S., Wang, T., and Tang, J.T., "Fuzzy Adaptive Output Tracking Control of Nonlinear Systems," Fuzzy Sets and Systems, Vol. 111, No. 2, pp. 169-182 (2000).

25. Wang, H.O., Tanaka, K., and Griffin, M.F., "An Approach to Fuzzy Control of Nonlinear System: Stability and Design Issues," IEEE Transactions on Fuzzy Systems, Vol. 4, No. 1, pp. 14-23 (1996).

26. Zheng, F., Wang, Q.G., and Lee, T.H., “Output Tracking Control of MIMO Fuzzy Nonlinear Systems Using Variable Structure Control Approach," IEEE Transactions on Fuzzy Systems, Vol. 10, No. 6, pp. 686-697 (2002). 